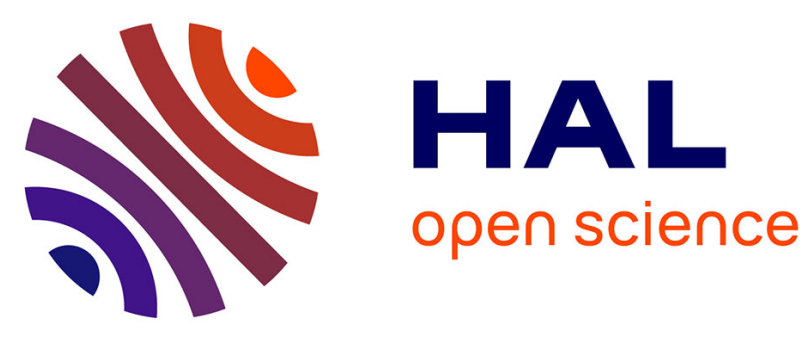

\title{
Supporting Semantic Search on Heterogeneous Semi-structured Documents
}

Yassine Mrabet, Nacéra Bennacer Seghouani, Nathalie Pernelle, Mouhamadou Thiam

\section{- To cite this version:}

Yassine Mrabet, Nacéra Bennacer Seghouani, Nathalie Pernelle, Mouhamadou Thiam. Supporting Semantic Search on Heterogeneous Semi-structured Documents. 22nd International Conference on Advanced Information Systems Engineering, CAISE 2010, Jun 2010, Hammamet, Tunisia. pp.224229, 10.1007/978-3-642-13094-6_18. hal-00502286

\section{HAL Id: hal-00502286 \\ https://hal.science/hal-00502286}

Submitted on 13 Jul 2010

HAL is a multi-disciplinary open access archive for the deposit and dissemination of scientific research documents, whether they are published or not. The documents may come from teaching and research institutions in France or abroad, or from public or private research centers.
L'archive ouverte pluridisciplinaire HAL, est destinée au dépôt et à la diffusion de documents scientifiques de niveau recherche, publiés ou non, émanant des établissements d'enseignement et de recherche français ou étrangers, des laboratoires publics ou privés. 


\title{
Supporting Semantic Search on Heterogeneous Semi-Structured Documents
}

\author{
Yassine Mrabet ${ }^{1,2}$, Nacéra Bennacer ${ }^{2}$, Nathalie Pernelle $^{1}$, \\ Mouhamadou Thiam ${ }^{1,2}$ \\ 1 LRI, Université Paris-Sud 11, INRIA Saclay, F-91893 Orsay cedex, France \\ \{Yassine.Mrabet, Nathalie.Pernelle, Mouhamadou.Thiam\}@lri.fr \\ 2 SUPELEC Systems Sciences (E3S), F-91192 Gif-sur-Yvette cedex, France \\ Nacera.Bennacer@supelec.fr
}

\begin{abstract}
This paper presents SHIRI-Querying ${ }^{3}$, an approach for semantic search on semi-structured documents. We propose a solution to tackle incompleteness and imprecision of semantic annotations of semistructured documents at querying time. We particularly introduce three elementary reformulations that rely on the notion of aggregation and on the document structure. We present the Dynamic Reformulation and Execution of Queries algorithm (DREQ) which combines these elementary transformations to construct reformulated queries w.r.t. a defined order relation. Experiments on two real datasets show that these reformulations greatly increase the recall and that returned answers are effectively ranked according to their precision.
\end{abstract}

\section{Introduction}

The research advances on automating ontology population and document annotation are promising. But even for named entity-based approaches $[1,2]$ or pattern-based approaches [5] it remains difficult to locate precisely instances since some of them may be blended in heterogeneous semi-structured documents. The granularity of the annotation could be precise, at the term level, or imprecise, at the node level, in a semi-structured document [5]. In the worst case, the annotated unit is the whole document. Semantic imprecision may also appear when associated annotations are not accurate enough (e.g. using Event metadata instead of Conference metadata). From another hand, annotations are often incomplete since automatic annotators do not find all instances and relations. To alleviate these problems, some semantic search systems try to gather answers satisfying the user query by going beyond the simple use of available metadata. Some approaches $[6,4]$ deal with semantic imprecision by approximating the concepts and the relations expressed in user queries using an ontology (e.g. exploiting subsomption, contextual closeness, path of semantic relations). Other works combine ontology-based search and classical keyword search $[7,3]$ in order to deal with incomplete annotations. The use of keywords increases the

\footnotetext{
${ }^{3}$ SHIRI : Digiteo labs project (LRI, SUPELEC)
} 
recall by retrieving instances that are not reachable using semantic annotations, but some semantic constraints of the query are relaxed.

In this paper, we propose an ontology-based search approach called SHIRIQuerying. Our contributions are: (i) a reformulation method to query incomplete and imprecise semantic annotations of semi-structured documents (ii) an order relation that ranks the constructed queries according to their relevance and (iii) a dynamic algorithm which builds and executes reformulated queries w.r.t. the defined order. The SHIRI-Querying system uses the standard W3C languages RDF/OWL for representing resources and SPARQL for their querying. It has two main components. The adapter is designed to conform the provided annotations to the SHIRI annotation model. It uses a set of logical rules and generates automatically the annotations base to be queried. The Query Engine processes ontology-based queries and reformulates them using the SHIRI annotation model. In this generic model [5] the granularity of the annotation is the document node (e.g. XML and HTML tags). Each node is annotated as containing one or several instances of different concepts of a given domain ontology. This allows bypassing the imprecise localisation of instances at the term level. The annotation model also allows representing structural links between document nodes, which enables dealing with the incompleteness of semantic relations in the provided annotations. We define three elementary query reformulations: the SetOfConcept and PartOfSpeech reformulations which allow retrieving instances that are aggregated in the same node and the neighborhood-based reformulation which allows retrieving instances located in close nodes that may be related by the required semantic relations. Reformulations of the user query are then obtained by combining these elementary transformations. The Dynamic Reformulation and Execution of Queries algorithm (DREQ) constructs these combinations and executes them w.r.t an order relation. This order relation gives priority to answers where nodes contain homogeneous instances and answers where nodes are linked by the required semantic relations. In contrast to most approaches which work on answers and/or whole annotated datasets, the answers are ranked as the reformulated queries are constructed. Experiments on two real datasets show that these reformulations greatly increase the recall and that the answers are effectively ranked according to their precision.

\section{Annotation Model}

Let $\mathcal{O}\left(\mathcal{C}_{\mathcal{O}}, \mathcal{R}_{\mathcal{O}}, \preceq, \mathcal{D}_{\mathcal{O}}\right)$ be the domain ontology where $\mathcal{C}_{\mathcal{O}}$ is the set of concepts, $\mathcal{R}_{\mathcal{O}}$ is the set of relations between concepts $\left(\mathcal{R}_{O}^{f}, \mathcal{R}_{O}^{i f}\right.$ are resp. functional and inverse functional relations), $\preceq$ denotes the subsumption relation between concepts or relations and $\mathcal{D}_{\mathcal{O}}$ defines the domain and the range for each relation. The annotation model, denoted $\mathcal{A}\left(\mathcal{C}_{\mathcal{A}}, \mathcal{R}_{\mathcal{A}}, \preceq, \mathcal{D}_{\mathcal{A}}\right)$, is generated automatically from the domain ontology. $\mathcal{C}_{\mathcal{A}}=\mathcal{C}_{\mathcal{O}} \cup \mathcal{C}_{\mathcal{S}}, \mathcal{R}_{\mathcal{A}}=\mathcal{R}_{\mathcal{O}} \cup \mathcal{R}_{\mathcal{S}} . \mathcal{C}_{\mathcal{S}}$ and $\mathcal{R}_{\mathcal{S}}$ are the concepts and the relations defined for the annotation task. In this model, concept instances are identified by URIs of document nodes and the literals associated by the has Value attribute are the textual contents of annotated nodes. 
We define the following aggregate metadata in $C_{S}$ and $R_{S}$ :

- The PartOfSpeech concept is used to annotate document nodes containing several instances of different concepts.

- The SetOfConcepts metadata is used to annotate document nodes containing several instances of the same concept. A concept $S e t O f c_{i}$ is defined as a subclass of SetOfConcepts for each concept $c_{i} \in \mathcal{O}$. Moreover, we define relations denoted $r$ Set and $r S e t^{-1}$ in $\mathcal{R}_{\mathcal{S}}$ derived from (inverse) functional relations $r$ in $\mathcal{R}_{\mathcal{O}}$ in order to represent relations between an instance and a set of instances.

- The neighborOf relation expresses a path in a XML/HTML document tree.

Instances of these metadata are generated by the adapter using a set of logical rules. If a document node contains only one instance of a domain concept $c$, it is annotated by $c$. The datatype properties of this instance become properties of the node. Else, it is annotated either by $\operatorname{SetOf}_{i}$ metadata or PartOfSpeech metadata. The property isIndexedBy is instantiated for PartOfSpeech nodes. The provided annotations of domain relations $r \in \mathcal{R}_{\mathcal{O}}$ are instantiated between nodes whose types are in $\mathcal{C}_{\mathcal{O}}$ (domain concepts). In the case where $r$ links a node of type $c_{j} \in \mathcal{C}_{\mathcal{O}}$ with a node of type $\operatorname{Set} O f c_{i}, r$ is substituted by $r S e t$ or $r S e t^{-1}$.

\section{Query Reformulations}

Preliminary Definitions: Consider the pairwise disjoint infinite sets $I, B, L$ and $V$ (IRIs, Blank nodes, Literals and Variables). A triple pattern is a triple $(s, p, o) \in(I \cup V) \times(I \cup V) \times(I \cup V \cup L)$. A basic graph pattern $P$ is a set of triple patterns. ? $v$ in a triple indicates that $v$ is a variable. An RDF query is a basic graph pattern or a constructed graph pattern (using constructors such as union or intersection). To facilitate the reading of this paper, we consider only queries described by basic graph patterns. The filters that we consider use equality and inclusion operators between variables and literal values.

We define a model-based query $q$ as a quadruplet $(P, S, F, D)$ where :

- $P$ is a basic graph pattern which complies with a model (i.e. $\mathcal{O}$ or $\mathcal{A}$ ). $V(P)$ denotes the set of variables of $P$ and $C(P)$ denotes the set of concepts of $P$.

$-F$ is a constraint defined by a logical combination of boolean-valued expressions.

$-S$ is the set of variables that appear in the $S E L E C T$ clause of the query.

- $D$ is an $\mathcal{A}$-compliant $\mathrm{RDF}$ dataset to be matched with $P$ and $F$.

Example: The $\mathcal{O}$-based query $q_{0}$ is defined by $\left(P_{0}, F_{0}, S_{0}, D\right)$ where : $P_{0}=\{$ (?art, rdf:type, Article), (?aut, rdf:type, Person), (?aut, hasName, ?aName), (?conf, rdf:type, Conference), (?art, publishedIn, ?conf)

(?art, authoredBy, ?aut), (?conf, hasName, ?cName)\}

$F_{0}:\{? c N a m e=" W W W 2008 "\}$ and $S_{0}:\{$ ?art, ?aut, ?aName $\}$

Neighborhood-based Reformulation: The aim of the neighborhood-based reformulation is to exploit the structural neighborhood of document nodes in 
order to find nodes that may be related by the semantic relations expressed in the user query. The neighborhood-based reformulation, denoted $f_{n r}$, substitutes the ontological relation of a given triple by a neighbor $O f$ relation.

Example : $f_{n r}\left(q_{0},(?\right.$ art, authoredBy, ?aut $\left.)\right)=q_{1}^{\prime}\left(P_{1}^{\prime}, F_{0}, S_{0}, D\right)$ where $P_{1}^{\prime}=$ $\left\{\right.$ (?art, rdf:type, Article), ...(?art, neighborOf, ?aut), ... \}. Applying $f_{n r}$ may generate semantically-independent subgraph patterns.

Definition 1. $p$ is a semantically-independent subgraph pattern of $P$ if : $-\forall v_{1}, v_{2} \in V(p),\left(? v_{1}, r, ? v_{2}\right) \in P \rightarrow\left(? v_{1}, r, ? v_{2}\right) \in p$

$-\forall\left(? v_{1}, r, ? v_{2}\right) \in P$ s.t. $v_{1}\left(\right.$ resp. $\left.v_{2}\right) \in V(p), v_{2}\left(\right.$ resp. $\left.v_{1}\right) \notin V(p) \rightarrow r=$ neighbor $O f$ Splitting a query into semantically-independent subgraph patterns allows applying distinct aggregative reformulations on distinct sub-parts of a query.

PartOfSpeech Reformulation: The PartOfSpeech reformulation denoted $f_{p r}$ assumes that the required semantic relations can be found between instances aggregated in the same node. It is applied on semantically-independent subgraph patterns. The target subgraph must be $\mathcal{O}$-based, i.e. it does not contain metadata from $C_{S}$ or $R_{S}$ (this constraint is part of the reformulations construction plan). $f_{p r}$ substitutes all filter constraints of the subgraph pattern by filter constraints on the textual contents of PartOfSpeech nodes. Equality constraints are relaxed into inclusion constraints.

Example: for $p \in P_{1}^{\prime}$ s.t. $p=\{(?$ art, rdf:type, Article $),(?$ conf, rdf:type, Conference), (?conf, hasName, ?cName), (?art, publishedIn, ?conf)\}, $f_{p r}\left(q_{1}^{\prime}, p\right)=q_{2}^{\prime}\left(P_{2}^{\prime}, F_{2}^{\prime}, S_{2}^{\prime}, D\right)$ s.t.

$P_{2}^{\prime}=\{($ ?pos, rdf:type, PartOf Speech $),(? p o s$, isIndexedBy, Conference), (?pos, isIndexedBy, Article), (?pos, hasValue, ?lPos), (?pos, neighborOf, ?aut), (?aut, rdf:type, Person), (?aut, hasName, ?aName) $\}$

$F_{2}^{\prime}:\left\{(? l P o s\right.$ contains "WWW2008") $\}, S_{2}^{\prime}:\{?$ aut, ?aName, ?pos $\}$

SetOfConcept Reformulation: The SetOfConcept reformulation, denoted $f_{s r}$, substitutes the ontological type $c$ of a given variable $v$ by the set $O f c$ type if for all triples of $P$ : (1) if $v$ is the subject, the relation $r$ is not inverse functional and (2) if $v$ is the object, the relation is not functional. The relation $r$ is then substituted by $r \mathrm{Set}^{-1}$ (case 1) or $r$ Set (case 2).

Example: $f_{s r}\left(q_{0}, ? a u t\right)=q_{3}^{\prime}\left(P_{3}^{\prime}, F_{0}, S_{0}, D\right)$ s.t. $P_{3}^{\prime}=\{($ ?art, rdf:type, Article), (?aut, rdf:type, SetOf Persons), (?conf, rdf:type, Conference), (?art, publishedIn, ?conf), (?art, authoredBySet, ?aut), (?aut, hasValue, ?aName), (?conf, hasName, ?cName)\}

Reformulations Construction Plan: The reformulation of a query $q_{0}\left(P_{0}\right.$, $\left.F_{0}, S_{0}, D\right)$ is a query $q_{i}\left(P_{i}, F_{i}, S_{i}, D\right)$ obtained by the composition of elementary PartOfSpeech, SetOfConcept and neighborhood-based reformulations. We consider that a set of document nodes is more relevant if its nodes do not contain aggregated instances and if they are related by the expected semantic relations. 
Definition 2. Let $N(q), \operatorname{Pos}(q)$ and Sets $(q)$ be resp. the number of neighborOf, PartOf Speech and SetOfc metadata in a query q. The order $\preccurlyeq$ is defined s.t. $q_{i} \preccurlyeq q_{j} \leftrightarrow\left(\left(N\left(q_{i}\right)>N\left(q_{j}\right)\right) \vee\left(\left(N\left(q_{i}\right)=N\left(q_{j}\right)\right) \wedge\left(\left(\operatorname{Pos}\left(q_{i}\right)>\operatorname{Pos}\left(q_{j}\right)\right) \vee\right.\right.\right.$ $\left.\left(\left(\operatorname{Pos}\left(q_{i}\right)=\operatorname{Pos}\left(q_{j}\right)\right) \wedge\left(\operatorname{Sets}\left(q_{i}\right) \geq \operatorname{Sets}\left(q_{j}\right)\right)\right)\right)$

Dynamic Reformulation and Execution of Queries Algorithm (DREQ) DREQ allows constructing and executing the reformulated queries with respect to $\preccurlyeq$. When $D R E Q$ is stopped at a given order, the answers are those retrieved by the best constructed queries. $D R E Q$ computes all reformulations in EXPTIME w.r.t the number of variables of the user query, but, as the algorithm is dynamic, we obtain a new set of equally-ordered reformulations in PTIME.

\section{Experimental Results}

SHIRI-Querying has been implemented and experimented to study how the precision and the recall measures vary according to the order relation. The neighborOf relation is defined as an undirected path of length $d$ in the HTML /XML tree. We also study how $d$ influences the results. The reformulations proposed in our approach can introduce wrong answers which may appear when the query is reformulated using $f_{s r}$ and $f_{p r}$ which relax filters in PartOfSpeech or SetOfConcepts nodes or using $f_{p r}$ and $f_{n r}$ which relax semantic relations. The two experimented datasets belong to the scientific conferences and publications domain.

The first dataset is composed of annotated publication references provided by the DBLP XML repository, the INRIA HTML server and the HAL XML repository. It consists of more than 10.000 RDF triples describing 1000 publications. We submitted a set of queries looking for conferences, their dates, locations, papers and authors. A precision of $100 \%$ and a recall of $100 \%$ were reached with an order threshold of 9 and $d \leq 3$. A smaller order threshold leads to a smaller recall and a higher distance $d$ leads to almost $0 \%$ precision. In this case $(d>3)$, in two data sources, each paper is associated to all conferences. The $100 \%$ values for the recall and the precision measures are due to the regular structure of the data sources. However, each data source has a different and specific structuring and the $D R E Q$ reformulations were able to integrate answers from all sources.

The second corpus consists of RDF annotations of 32 call-for-papers web sites and is consequently very heterogeneous. These annotations (consisting of 30.000 RDF triples) were generated automatically using SHIRI-Extract [5]. We then submitted a set of 15 queries. Without reformulation, all queries have no answers (0\% recall), while we obtained a $56 \%$ recall by using the $D R E Q$ algorithm for $d$ $\leq 7$. At the same distance threshold $(d \leq 7)$ the precision is still $72 \%$. The results show that domain relations can often be retrieved between instances located in close document nodes. Figure 1(b) presents the average precision value for the same set of user queries, for several values of $d$, by varying the order threshold from 1 to 18. The precision variations show that the order relation is relevant to rank the answers. 


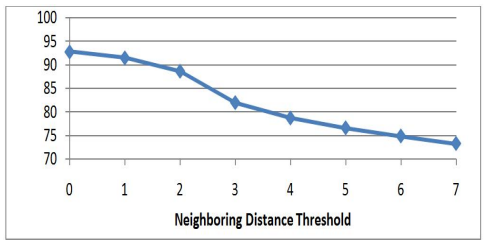

(a) Precision according to d

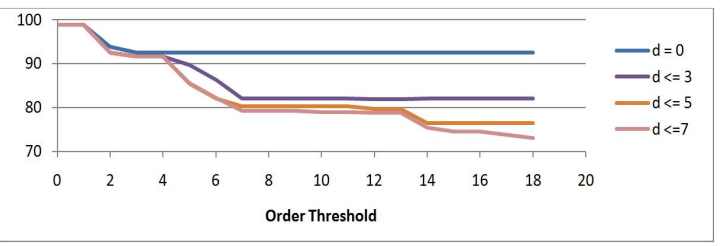

(b) Precision according to order

Fig. 1. Answers' Precision

\section{Conclusion and Future Work}

In this paper, we presented the SHIRI-Querying approach to support semantic search on heterogeneous semi-structured documents. Ontology-based user queries are reformulated to gather document nodes from documents that were annotated in an imprecise and incomplete manner by semantic annotation tools. These reformulations allow retrieving instances that are related by the requested semantic relations even if these relations are not available in the knowledge base. We defined an order relation between reformulated queries to give priority to queries that preserve most the semantics of the user query. All reformulations are constructed dynamically w.r.t this order relation in the $D R E Q$ algorithm. Experimental results show that the recall greatly increases and that the precision decreases reasonably as the ordered reformulated queries are performed. In the near future we plan to combine keyword-based search with our reformulation approach to increase the recall without losing the semantics of the query. We also plan to use semantic-based heuristics exploiting functional properties of relations in order to avoid some wrong answer cases.

\section{References}

1. Borislav P., Atanas K., Angel K., Dimitar M., Damyan O., Miroslav G.: KIM Semantic Annotation Platform. J. of Nat. Lang. Engineering 10(3-4), 375-392, 2004.

2. Etzioni O., Cafarella M., Downey D., Kok S., Popescu A., Shaked T., Soderland S., Weld D., and Yates A.: Unsupervised named-entity extraction from the web: An experimental study. Artificial Intelligence, 165(1): 91-134, 2005.

3. Bhagdev R., S. Chapman, F. Ciravegna, V. Lanfranchi and D. Petrelli.: Hybrid Serach: Effectively Combining Keywords and Semantic Searches. ESWC, 2008.

4. Corby O., Dieng-Kuntz R., Gandon F. and Faron-Zucker C.: Searching the semantic web : Approximate query processing based on ontologies. IEEE Intelligent Systems Journal, Computer Society. Vol. 21, n. 1, pp 20-27, 2006.

5. Thiam, M., Bennacer, N., Pernelle, N. and Lo, M.: Incremental Ontology-Based Extraction and Alignment in Semi-Structured Documents. DEXA, 2009.

6. Hurtado C-A., Poulovassilis A. and Wood, P-T.: A Relaxed Approach to RDF Querying. International Semantic Web Conference, ISWC, 2006.

7. Castells, P. Fernàndez, M. Vallet, D.: An adaptation of the vector-space model for ontology-based information retreival. IEEE T. on Know. and Data Eng. 19(2), 2007. 